\title{
A Fully Anonymous Electronic Payment Scheme for B2B*
}

\author{
Josep Lluís Ferrer-Gomila, Magdalena Payeras-Capellà, and Ll. Huguet-Rotger \\ Universitat de les Illes Balears \\ Carretera de Valldemossa km. 7.5, Palma de Mallorca, 07122, Spain \\ \{dijjfg, mpayeras, dmilhr0\}@clust.uib.es
}

\begin{abstract}
In this paper we present a fully anonymous (for payer and payee) electronic payment scheme for B2B transactions involving high amounts, without using tamper resistant devices, without pseudonyms, and preventing double spending. Schemes with detection of double spending at deposit are not well suited for high amount transactions (merchants and banks don't want to assume potential high losses). Our scheme allows anonymity revocation in case of illegal activities (using prevention techniques).
\end{abstract}

\section{Introduction}

B2B transactions demand a specific electronic payment scheme, because amounts can be high (more than 1000 dollars). It should be "impossible" to counterfeit money. It should not be based on tamper resistant devices [1,4] (an attack could be very attractive). Nobody wants to assume possible double spending (with high amounts). So, it is not useful to detect double spending a posteriori, and we can not use transferable [5, 9] and/or off-line [2, 3, 7, 8] schemes. The anonymity should be absolute (as in paper-based transactions, e.g., for undeclared earnings). Otherwise, merchants will continue using paper money. We have to achieve the untraceabilty property (not achieved in $[1,6])$. We don't want to use pseudonyms because it is not a real anonymity. The behavior of the bank has to be verifiable. Merchants and the bank have the necessary communications infrastructure to carry out on-line transactions. Finally, anonymity should be revocable if the appropriate authorities order it. In fact, some of the previous requirements were listed in [9]. But we have to keep in mind that this electronic payment scheme is specific for B2B and for high amounts.

Here we present a scheme that avoids counterfeiting, forgery and double spending, without using tamper resistant devices (nobody assumes undesirable risks). Untraceability and anonymity are guaranteed without using pseudonyms. Finally, our scheme allows the anonymity revocation in case of illegal activities (using prevention techniques when the illegal activity is suspected).

\footnotetext{
* This work has been partially supported by the Balearic Islands Government under project PRDIB-2002GC3-18.
} 


\section{Fully Anonymous Electronic Payment Scheme}

The presented scheme includes withdrawal, transfer and deposit sub-protocols. We use the term $e$-note to refer to the electronic bank notes. In the description of the protocol $A$ is a merchant acting as a payer, $B$ is the payee and $F$ is the bank. Other notation and elements are as follows:

$\begin{array}{ll}\mathrm{ID}_{\mathrm{X}} & \text { identity of actor } X \\ \mathrm{Q}_{\mathrm{i}} & \text { amount to be withdrawn, transferred or deposited } \\ \mathrm{Y}, \mathrm{Z} & \text { concatenation of two messages (or tokens) } Y \text { and } Z \\ \text { Sign } & \text { digital signature of principal } i \text { on message } Y \\ \mathrm{i} \rightarrow \mathrm{j}: \mathrm{Y} & \text { principal } i \text { sends message (or token) } Y \text { to principal } j \\ \mathrm{E}_{\mathrm{K}}(\mathrm{M}) & \text { symmetric encryption of message } M \text { with key } K \\ \mathrm{PU}_{\mathrm{F}}(\mathrm{K}) & \text { key } K \text { enciphered with the public key of the bank } \\ \mathrm{SN}_{\mathrm{i}}=\mathrm{H}\left(\mathrm{SP}_{\mathrm{i}}\right) & \text { serial number }\left(S N_{i}\right) \text { of an } e \text {-note, hash of a random secret proof }\left(S P_{i}\right) \\ \mathrm{M}_{\mathrm{i}}=\operatorname{Sign}_{\mathrm{Fx}}\left(\mathrm{SN}_{\mathrm{i}}\right) & \text { signature on } S N_{i} \text { with a private key indicating value } x\end{array}$

\subsection{Withdrawal Sub-protocol}

In the withdrawal sub-protocol, a merchant, $A$, requests an $e$-note to $F$. $F$ creates an $e$ note and debits $A$ 's account:

$$
\begin{array}{ll}
\text { 1. } \mathrm{A} \rightarrow \mathrm{F}: & \mathrm{ID}_{\mathrm{A}}, \mathrm{Q}_{1}, \mathrm{SN}_{1}, \operatorname{Sign}_{\mathrm{A}}\left(\mathrm{Q}_{1}, \mathrm{SN}_{1}\right) \\
\text { 2. } \mathrm{F} \rightarrow \mathrm{A}: & \mathrm{Q}_{1}, \mathrm{SN}_{1}, \mathrm{M}_{1}
\end{array}
$$

$A$ generates a random number $S P_{l}$ (the secret proof to validate the e-note), and it must be kept secret. $S N_{l}$ (the serial number of the future e-note) is a hashing of $S P_{l}$. A proves ownership of his account signing the serial number and the amount, $Q_{1}$. F's signature on $S N_{l}$, and $S P_{l}$, is the e-note. $S N_{l}$ will be used to prevent double spending of the $e$-note. A can prove the ownership with the knowledge of $S P_{l}$ and $M_{l}$.

To redeem an $e$-note, the owner must show the knowledge of the $e$-note secret proof $\left(S P_{l}\right)$, but he is not forced to reveal his identity. If $F$ saves all available information about the e-note, it could recognise that $e$-note at deposit, but thanks to the use of the transfer sub-protocol (see section 2.2), the bank (or the collusion of the bank and the merchant depositing the e-note) cannot reveal where $A$ spent it. So payments will be anonymous and untraceables. And the scheme is secure against money forging.

\subsection{Transfer Sub-protocol}

When $A$ wants to pay to $B, A$ executes the following sub-protocol:

1. A $\rightarrow$ B: Purchase_order

2. B $\rightarrow$ A: $\quad \mathrm{Q}_{\mathrm{i}}, \mathrm{SN}_{\mathrm{i}}, \operatorname{Sign}_{\mathrm{B}}\left(\mathrm{Q}_{\mathrm{i}}, \mathrm{SN}_{\mathrm{i}}\right)$

3. $\mathrm{A} \rightarrow \mathrm{F}: \quad \mathrm{PU}_{\mathrm{F}}(\mathrm{K}), \mathrm{E}_{\mathrm{K}}\left(\mathrm{SN}_{\mathrm{j}}, \mathrm{M}_{\mathrm{j}}, \mathrm{SP}_{\mathrm{j}}, \mathrm{Q}_{\mathrm{i}}, \mathrm{SN}_{\mathrm{i}}, \mathrm{SN}_{\mathrm{r}}\right)$

4. $\mathrm{F} \rightarrow \mathrm{A}: \quad \mathrm{M}_{\mathrm{i}}, \mathrm{M}_{\mathrm{r}}$

5. $\mathrm{A} \rightarrow \mathrm{B}: \quad \mathrm{SN}_{\mathrm{i}}, \mathrm{M}_{\mathrm{i}}$ 
$B$ sends to $A$ a serial number $\left(S N_{i}\right)$, the price of the item $\left(Q_{i}\right)$ and the digital signature of the previous information, as a response to the Purchase_order, without revealing the secret proof $\left(S P_{i}\right) . A$ will request to $F$ an $e$-note to pay $B$, with the serial number given by $B\left(S N_{i}\right)$. A sends her $e$-note $M_{j}$ to the bank, with the associated secret proof $\left(S P_{j}\right)$. The request is encrypted with a session key $(K)$, so nobody can intercept $S P_{j}$. $A$ indicates the amount $\left(Q_{i}\right)$ of the $e$-note $M_{j}$ to be converted in the new $e$-note using $S N_{i}$. The remaining fraction $Q_{r}$ (if $Q_{i}<Q_{j}$ ) will be used to create another $e$-note with serial number $S N_{r}$. $F$ cannot find out the identities of the merchants.

If $S N_{j}$ is found in the list of spent $e$-notes, $F$ has detected a double spending attempt, and will abort the operation. If the $e$-note $\left(S P_{j}, M_{j}\right)$ is valid, $F$ creates the new $e$-notes $M_{i}$ and $M_{r}$, and sends them to $A$. $A$ knows the $e$-note $M_{i}$ and $S N_{i}$, but $A$ doesn't know $S P_{i}$. A stores the information related to the payment during an established period. This information can be requested in case of anonymity revocation. The scheme is anonymous for $A$, because $B$ doesn't know the identity of $A$.

$B$ checks the validity of the $e$-note $M_{i}$ (verifying the signature of $F$ ). Only $B$ knows $S P_{i}$ and he is the only one that can spend that $e$-note. He doesn't need to contact $F$. Now, $B$ has an $e$-note with the same properties that a withdrawn one. $B$ can deposit it identifying his account. Also, $B$ can use the $e$-note for a new payment, but a collusion between $A$ and $F$ will be able to trace $B$. To solve this problem $B$ has to use the autotransfer sub-protocol.

Transfer Sub-protocol Applied to Auto-transfer. $A$ knows $S N_{i}$ and $B$ 's identity. So, payments with that $e$-note could be traced by the collusion of $A$ and $F$. The solution is the auto-transfer operation:

1. $\mathrm{B} \rightarrow \mathrm{F}: \quad \mathrm{PU}_{\mathrm{F}}(\mathrm{K}), \mathrm{E}_{\mathrm{K}}\left(\mathrm{SN}_{\mathrm{i}}, \mathrm{M}_{\mathrm{i}}, \mathrm{SP}_{\mathrm{i}}, \mathrm{Q}_{\mathrm{s}}, \mathrm{SN}_{\mathrm{s}}, \mathrm{SN}_{\mathrm{t}}\right)$

2. $\mathrm{F} \rightarrow \mathrm{B}: \quad \mathrm{M}_{\mathrm{s}}, \mathrm{M}_{\mathrm{t}}$

$B$ calculates $S N_{s}$ and $S N_{t}$ from the random secret proofs $S P_{s}$ and $S P_{t}$, respectively. $B$ requests $F$ that a specific $e$-note is going to be transferred. $B$ sends $S P_{i}$ encrypted with a session key (and other information analogous to the previous case).

If the $e$-note is valid (e.g., not double spent), $F$ creates two new e-notes with the new serial numbers and the required values, and $S P_{i}$ is appended to the list of spent $e$ notes. $F$ doesn't know who is the user auto-transferring the e-note. Furthermore, $F$ cannot distinguish if the user is auto-transferring the total amount of the e-note, or if he is preparing a payment with a fraction of the e-note and auto-transferring the remaining part, or if he is preparing two payments.

\subsection{Deposit Sub-protocol}

In the deposit sub-protocol, it is necessary an identification of the merchant's account:

$$
\text { 1. } \mathrm{B} \rightarrow \mathrm{F}: \quad \mathrm{PU}_{\mathrm{F}}(\mathrm{K}), \mathrm{E}_{\mathrm{K}}\left(\mathrm{ID}_{\mathrm{B}}, \mathrm{SN}_{\mathrm{i}}, \mathrm{M}_{\mathrm{i}}, \mathrm{SP}_{\mathrm{i}}, \mathrm{Q}_{\mathrm{i}}\right), \operatorname{Sign}_{\mathrm{B}}\left(\mathrm{ID}_{\mathrm{B}}, \mathrm{SN}_{\mathrm{i}}, \mathrm{M}_{\mathrm{i}}, \mathrm{SP}_{\mathrm{i}}, \mathrm{Q}_{\mathrm{i}}\right)
$$

$B$ sends the secret proof $S P_{i}$, and some identifying information (to deposit money in the right account), all encrypted with a session key $K$. $F$ checks the validity of the $e$ note, and if it is correct then credits B's account. 


\section{Conclusion: Fraud Protection and Privacy}

We have achieved security requirements: e-notes cannot be counterfeited (thanks to the use of the bank private keys), overspending and double spending are avoided ( $e$ notes are created after a debit in an user account, and the bank saves the list of redeemed serial numbers, deposited and transferred, with their secret proofs), and stolen $e$-notes cannot be redeemed (it is necessary the secret proof, and it is encrypted when is transmitted). On the other hand, our scheme provides anonymity and untraceability to payers and payees, thanks to the auto-transfer subprotocol. E-notes can be transferred multiple times without depositing and without any identification. Payments between the same pair of merchants are unlinkable. There isn't any relationship between them: new serial numbers are used in each payment.

Our scheme prevents illegal activities (as blackmailing, money laundering and illegal purchases/sales). For example, if blackmailing is suspected or reported, the appropriate authority will allow to the bank to demand the identity of the user who will try to transfer or deposit the suspicious serial number $(S N)$. We don't use blind signatures to achieve anonymity, and so a blackmailed user always knows the serial number of the money given to the blackmailer. If money laundering is suspected, the authority will allow to the bank to demand user identification when this user is going to transfer the money.

We want to remark that we don't use tamper resistant devices, nor pseudonyms. The possibility of anonymous payment and redemption, the double spending prevention and other security properties, makes this scheme suitable for anonymous payments of high amounts in $\mathrm{B} 2 \mathrm{~B}$ transactions.

\section{References}

[1] Anderson, R., et alter: "NetCard - a practical electronic cash system", 4th Security Protocols International Workshop, LNCS 1189, pages 49-57, Springer Verlag, 1996.

[2] Brands, S.: "Untraceable off-line cash in wallet with observers", Crypto'93, LNCS 773, pages 302-318, Springer Verlag, 1993.

[3] Chaum, D., et alter: "Untraceable electronic cash", Crypto'88, LNCS 403, pages 319-327, Springer Verlag, 1988.

[4] Chaum, D. and Pedersen, T.: "Wallet databases with observers", Crypto'92, LNCS 740, pages 89-105, Springer Verlag, 1992.

[5] Eng, T. and Okamoto, T.: "Single-term divisible electronic coins", Eurocrypt'94, LNCS 576, pages 306-319, Springer Verlag, 1994.

[6] Medvinsky, G. and Neuman, B.C.: "Netcash: A design for practical electronic currency on the internet", 1st ACM Conference on Computer and Communication Security, pages 102106, 1993.

[7] Mu, Y., et alter: "A Fair Electronic Cash Scheme”, ISEC 2001, LNCS 2040, pages 20-32, Springer Verlag, 2001.

[8] Nakanishi, T. and Sugiyama, Y.: "Unlinkable Divisible Electronic Cash", ISW 2000, LNCS 1975, pages 121-134, Springer Verlag, 2000.

[9] Okamoto, T. and Otha, K.: “Universal electronic cash”, Crypto'91, LNCS 576, pages 324337, Springer Verlag, 1991. 